\section{Case Reports in Ophthalmology}

\title{
Effective Intravitreal Injections of Bevacizumab in a Case of Serous Macular Detachment from the Superior Border of the Posterior Staphyloma
}

\author{
Yukiko Tsubota $^{a}$ Hidenori Takahashi, $^{a}$ Kenji Sugisaki ${ }^{a}$ \\ Tatsuro Tanabe $^{a}$ Yujiro Fujino ${ }^{a}$ \\ aJapan Community Healthcare Organization, Tokyo Shinjuku Medical Center, \\ Tokyo, Japan; ${ }^{b}$ Jichi Medical University, Tochigi, Japan
}

\section{Keywords}

Posterior staphyloma Serous retinal detachment $\cdot$ Tilted disk syndrome

\begin{abstract}
Purpose: We present an atypical case of submacular fluid leading to serous macular detachment. Method/Patient: A 69-year-old man was evaluated for metamorphopsia in the left eye. Results: Best-corrected visual acuity was $20 / 25$ in both eyes. He had undergone cataract surgeries in both eyes 12 years ago. The axial length was $25.93 \mathrm{~mm}(\mathrm{OD})$ and $24.12 \mathrm{~mm}(\mathrm{OS})$. Optical coherence tomography showed posterior staphylomas and subretinal fluid on the superior border of the staphylomas in both eyes; in the left eye, submacular fluid was noted extending up to the macula. Fundus fluorescein angiography revealed leakage from the superior border of the staphylomas in both eyes. The fluid persisted for 4 months. Four consecutive, monthly injections of bevacizumab $(1.25 \mathrm{mg} / 0.05 \mathrm{~mL})$ were administered in the left eye; subsequently, the subretinal fluid gradually dissipated from the macula and became localized at the superior border of the staphyloma. This localization persisted for 12 months. Conclusions: We have detailed a case of submacular fluid that spread from the superior
\end{abstract}




\section{Case Reports in Ophthalmology}

border of the posterior staphyloma in a patient with macular detachment, in whom intravitreal injections of bevacizumab were highly effective in eliminating the fluid.

(C) 2017 The Author(s)

Published by S. Karger AG, Basel

\section{Introduction}

In eyes with tilted disc syndrome (TDS), serous retinal detachment may develop in the macular region because the edge of an inferior staphyloma typically crosses the macular area [1]. Subretinal leakage in myopic eyes with a posterior staphyloma or TDS is a poorly recognized disease entity [2]. Currently, there is no effective treatment for serous macular detachment associated with TDS, although photocoagulation of accessible angiographically leaking areas has been assayed with variable outcomes [3]. On the other hand, intravitreal injection of the antiangiogenic drug bevacizumab has failed to show efficacy in recent reports $[4,5]$. Photodynamic therapy has also failed to show efficacy in a recent report [5]. We present our findings on a highly myopic patient with macular detachment that spread from the superior border of the posterior staphyloma who was effectively treated with intravitreal injections of bevacizumab.

\section{Case Report}

A 69-year-old man presented with metamorphopsia in his left eye. His medical history included hypertension, chronic sinusitis, and prostatic hypertrophy. His best-corrected visual acuity was 20/25 in both eyes. He had undergone cataract surgeries in both eyes 12 years ago. Both eyes showed mydriasis of only up to $3 \mathrm{~mm}$. The axial lengths were $25.93 \mathrm{~mm}$ OD and $24.12 \mathrm{~mm}$ OS. Fundus examination showed posterior staphyloma in both eyes, the border of which was located superior and temporal to the macula (Fig. 1a) in both eyes. Optical coherence tomography demonstrated that subretinal fluid (SRF) was located on the superior border of the staphyloma in both eyes; submacular fluid had spread to the macula in only the left eye without choroidal neovascularization or polypoidal lesions (Fig. 2). Fundus fluorescein angiography showed a belt-shaped area of granular hyperfluorescence corresponding to the border of the staphyloma in both eyes (Fig. 1b). The hypofluorescence was more pronounced in the early phase of indocyanine green angiography (Fig. 1c). The patient was prescribed kallidinogenase (150 units/day) for 4 months, but no response was noted. After obtaining informed consent, off-label bevacizumab (Avastin, $1.25 \mathrm{mg} / 0.05 \mathrm{~mL}$ ) was injected intravitreally at monthly intervals in the left eye; this procedure was performed 4 times.

At 4 months after the treatment, the SRF dissipated from the macula and became localized at the border of the staphyloma, and metamorphopsia had disappeared (Fig. 3). The SRF localization has persisted for 12 months.

\section{Discussion}

The clinical course of this patient suggested that intravitreal injections of bevacizumab for serous macular detachment were highly effective. Anti-VEGF agents have the following 2 effects: antiangiogenesis and inhibition of vascular permeability. Milani et al. [3] reported that intravitreal bevacizumab injections led to the disappearance of an early leaking point on 


\section{Case Reports in Ophthalmology}

\section{DOI: $10.1159 / 000453605$} www.karger.com/cop

Tsubota et al.: Effective Intravitreal Injections of Bevacizumab in a Case of Serous Macular Detachment from the Superior Border of the Posterior Staphyloma

fluorescein angiography, but optical coherence tomography did not reveal a reduction of serous fluid. In the present case, before intravitreal injections of bevacizumab, the SRF had not changed for 4 months. After 4 consecutive monthly injections of bevacizumab, the SRF decreased and the localization has persisted for 12 months but has not changed. Intravitreal injections of bevacizumab are thought to be effective in reducing choroidal permeability in this case.

In the present case, serous macular detachment occurred due to the spread of SRF from the superior border of the posterior staphyloma. Pardo-López et al. [4] reported that the edges of an inferior staphyloma usually cross the macular area. Typically, SRF with posterior staphyloma is observed when the macula is located on the border of the posterior staphyloma. We consider that it is only under these conditions that patients recognize the symptoms, and there are potentially many unrecognized cases of SRF on the staphyloma border far from the macula. Some reports have named this disease TDS $[3,6]$. We agree that in most eyes, the macula is located on the border of the posterior staphylomas, and the borders also cross the disc, leading to tilted disks. However, this is not a standardized name, and we consider that this clinical manifestation should be termed "serous retinal detachment on the border of the posterior staphyloma." The alterations of the retinal pigment epithelium and choroidal vascular network could be caused by a change in the curvature at the border of the staphyloma in highly myopic eyes even away from the macula [4]. The development of SRF might thus be related to retinal pigment epithelium dysfunction and/or choroidal permeability [6].

In summary, we have detailed a case of serous macular detachment from the superior border of the posterior staphyloma, in whom intravitreal bevacizumab therapy was used for efficacious and safe treatment. Since this finding is only supported by a single case report, it would be desirable to accumulate further data and figures to demonstrate the potential safety and efficacy of bevacizumab for this condition.

\section{Disclosure Statement}

Y.T. and K.S. have no conflicts of interest. H.T. received lecturer's fees from Kowa Pharmaceutical, Novartis Pharmaceuticals, Bayer Yakuhin, and Santen Pharmaceutical; an educational presentation fee from Tochigi Prefectural Ophthalmologists Association, and grants from Novartis Pharma outside this work. T.T. received lecturer's fees from Alcon Japan Ltd. outside this work. Y.F. received lecturer's fees from Alcon Japan Ltd. and Otsuka Pharmaceutical and an educational presentation fee from Tokyo Association of Ophthalmologists outside this work.

\section{Statement of Ethics}

Institutional review board approval for this therapy was obtained from the Japan Community Healthcare Organization, Tokyo Shinjuku Medical Center. Informed consent was obtained from the patient. The protocol adhered to the tenets of the Declaration of Helsinki. 
Tsubota et al.: Effective Intravitreal Injections of Bevacizumab in a Case of Serous Macular Detachment from the Superior Border of the Posterior Staphyloma

\section{References}

$\checkmark 1$ Ohno-Matsui K, Shimada N, Moriyama M, Tokoro T, Mochizuki M: A case of chronic central serous chorioretinopathy on the border of a posterior staphyloma in an eye with pathologic myopia. Jpn J Ophthalmol 2009;53:282-284.

-2 Leys AM, Cohen SY: Subretinal leakage in myopic eyes with a posterior staphyloma or tilted disk syndrome. Retina 2002;22:659-665.

-3 Milani P, Pece A, Pierro K, Seidenari P, Radice P, Scialdone A: Bevacizumab for macular serous neuroretinal detachment in tilted disk syndrome. J Ophthalmol 2010;2010:970580.

-4 Pardo-López D, Gallego-Pinazo R, Mateo C, Rohrweck S, Suelves AM, Dolz-Marco R, Mullor JL, DíazLlopis M: Serous macular detachment associated with dome-shaped macula and tilted disc. Case Rep Ophthalmol 2011;2:111-115.

5 Donati MC, Miele A, Abbruzzese G, Giuntoli M, Giansanti F, Menchini U: Treatment of macular serous neuroretinal detachment in tilted disk syndrome: report of 3 cases. Eur J Ophthalmol 2013;23:267270.

6 Hirano Y, Yasukawa T, Tsukada A, Yokoyama S, Ito Y, Nakazawa Y: Resolution of exudative changes refractory to ranibizumab after aflibercept injections at the margin of inferior staphyloma in tilted disc syndrome. Ophthalmic Surg Lasers Imaging Retina 2015;46:384-386.
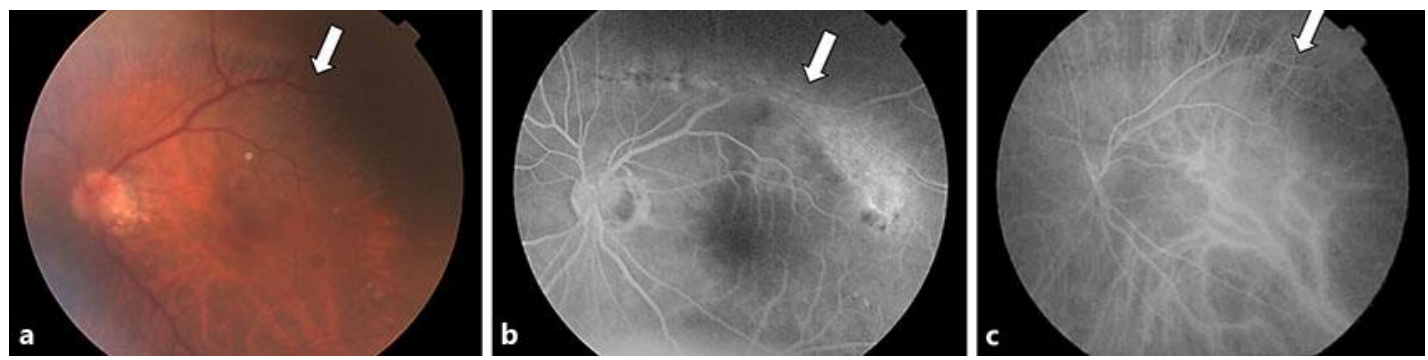

Fig. 1. Fundus photograph of the left eye showing myopic disc and posterior staphyloma. The superior border of the staphyloma was located far from the macula (a). Fluorescein angiogram showing a beltshaped area of granular hyperfluorescence corresponding to the superior border of the posterior staphyloma (b). Indocyanine green angiogram showing hypofluorescence due to choriocapillaris occlusion at the superior border of the staphyloma (c). 


\section{Case Reports in Ophthalmology}
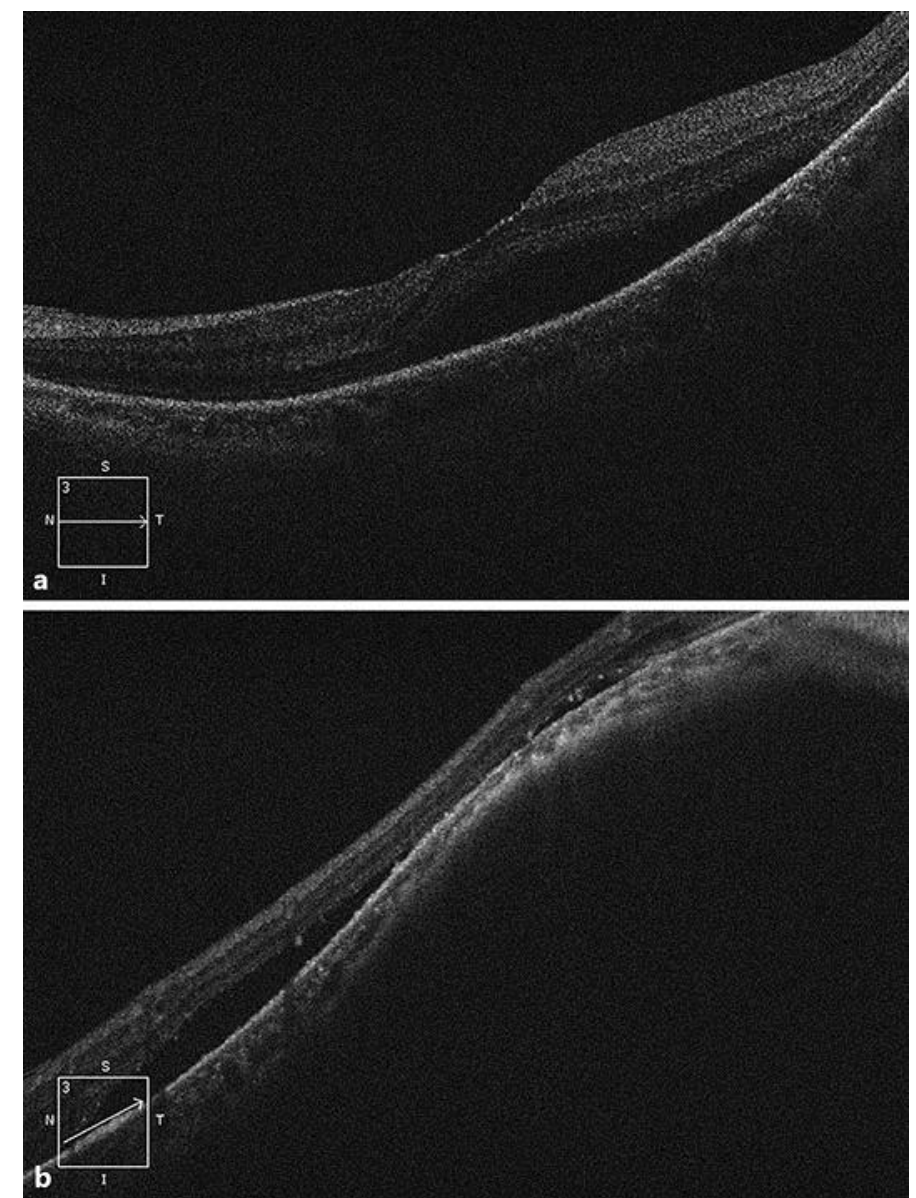

Fig. 2. Before bevacizumab treatment in the left eye, horizontal cross-sectional optical coherence tomography images revealed submacular fluid (a), and a cross-sectional optical coherence tomography image of both the macula and the superior border of the posterior staphyloma revealed the consecutive presence of subretinal fluid spreading from the superior border of the posterior staphyloma to the macula (b). 


\section{Case Reports in Ophthalmology} www.karger.com/cop

Tsubota et al.: Effective Intravitreal Injections of Bevacizumab in a Case of Serous Macular Detachment from the Superior Border of the Posterior Staphyloma
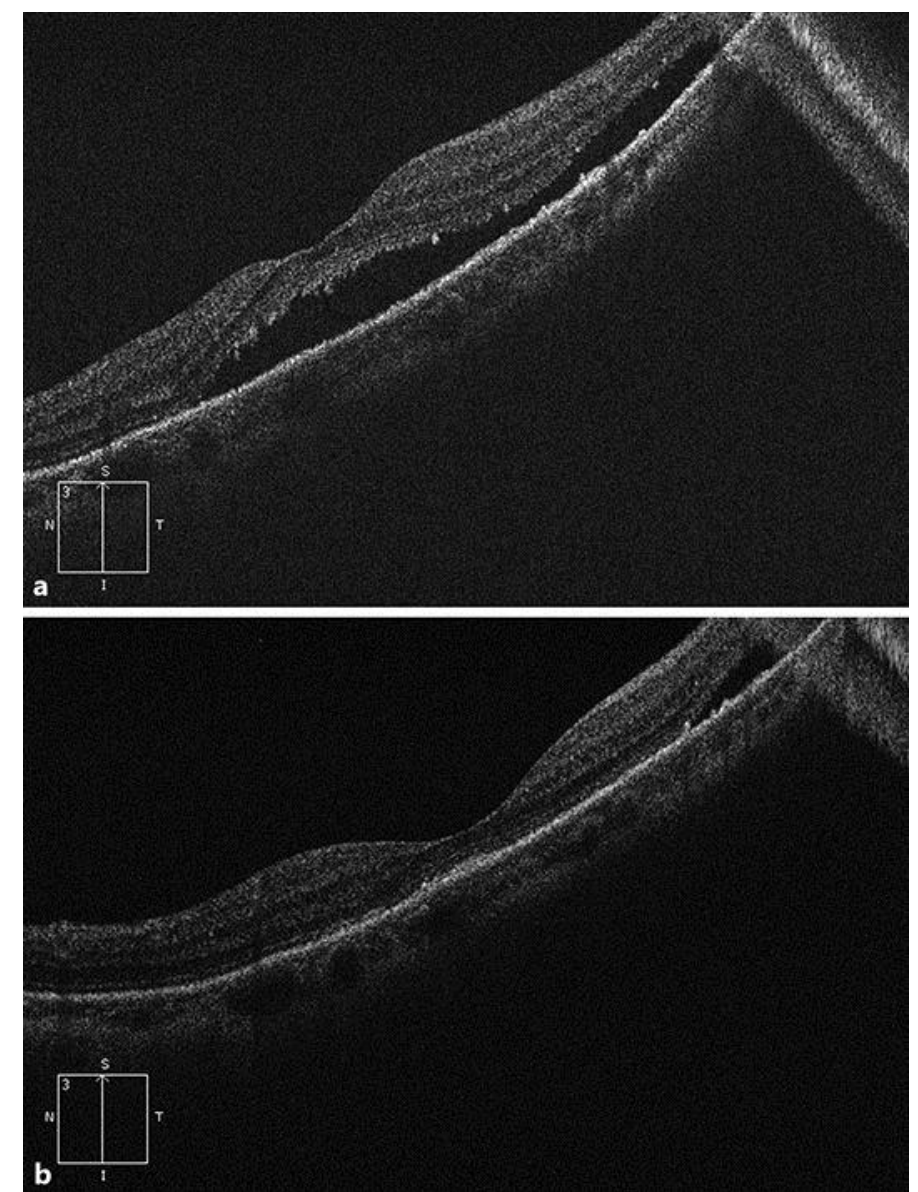

Fig. 3. After a single intravitreal bevacizumab injection, the serous macular detachment was improved (a). After 4 consecutive monthly intravitreal bevacizumab injections, the serous macular detachment resolved (b), and SRF became localized to the superior border of the staphyloma and persisted for 12 months. 